\title{
Expanding health systems for sustainable development
}

more expansive and interrogative approach to creating systems for better health pushing politicians to seek ways of collaborating across multiple sectors of government, urging private businesses and citizens to reconsider their roles, responsibilities and reasons for contributing to public health, stimluating funders to support research across traditional disciplines, inspiring scientists to step beyond the security of familiar methods and procedures, and encouraging ethicists to consider which arguments for equity, rights and fariness do and do not work in different settings. velopment Goals (MDGs, 2000-15) which focused on maternal and child health and major communicable diseases. But the greater ambition of the 2030 Agenda is to anchor heath in development, recognizing that good health depends on and contributes to other development goals, underpinning social justice, economic prosperity, and environmental protection. These aspirations have been frequently voiced but scarcely pursued, and the SDGs are often treated simply as a checklist of new goals and targets. And yet their potential is far greater collectively they should be a force for the discovery of new ways to achieve better health and well-being. To this end, the legacy of the MDGs, and the structure of the SDGs, lead to a testable proposition for research: advance health and development by expanding the scope and enhancing the effectiveness of the systems and services that prevent and treat illness. At stake is the question of how to accelerate gains in health through broad-based sustainable development (SDGs), building on the successes and compensating for the weaknesses of targeted, time-limited health programs (MDGs).

That question in not new, and neither is the view that systems analysis can help answer it. The idea of strengthening health systems has been pursued for years in some segments of the global research and development community, but has usually been tackled within separate compartments of the health sector. The SDGs promote a

World Health Organization, Geneva, Switzerland. Email: chrisdye56@gmail.com sources disproportionately, or simply through neglect (4). Re-integrating disease control programs into general health services now poses a series of well-defined operational challenges (5-7).

Second, these studies reaffirm the indirect health risks (and potential benefits) of social, economic and environmental factors that lie outside the control of the health sector, such as female education and fertility, family income, and access to safe water and sanitation. The idea of modifying these factors by working across disciplines and sectors has a long history, but multidisciplinary research and intersectoral action are still exceptional rather than routine. For example, cross-disciplinary initiatives from the human ("Health in All Policies", HiAP) and veterinary health ("One Health") communities have common aims but remain largely separate enterprises. One challenge of working across sectors is to balance the control of selected diseases of high importance (such as HIV/AIDS, tuberculosis, malaria) with the management of systemic risks to health (e.g. provision of safe water, sanitation and housing, or mechanisms for finance, governance, monitoring and planning). It is clear that both are important, but the MDGs emphasized the former while the SDGs stress the latter.

The choice between the two should encourage, in the SDG era, a more openminded approach to investigating causes and risks of ill health, unconstrained by specific methods or disciplines. The ideal response to any risk would consider interventions across the whole chain of events from primary (upstream) causes to ultimate (downstream) effects on health. But assessment of risk is typically partial, rarely comprehensive. Thus, if a study focuses, for example, on selected social determinants of tuberculosis (e.g. income, employment, housing), then the proposed solutions will address only these determinants, missing other possible behavioral (tobacco smoking), environmental (air pollution), medical (HIV coinfection), metabolic (diabetes) and occupational (mining) risks and, critically, the interactions between them. Furthermore, the failure to implement proven in- 
terventions (e.g. early diagnosis and treatment for TB) is rarely considered to be a "risk factor", but could be the largest modifiable cause of illness or death in any given setting (9). There is not yet a standard, comprehensive approach to evaluating preventable risks to health.

The MDGs became the world's principal framework for international cooperation in development. Now that the SDGs have inherited that role, advertising the interlinkages between health and all other goals, the debate about how to improve health is taking place, not just among health ministers, but also among heads of state, private businesses, non-government organizations and civil society. That national and international debate should be informed by a research agenda that is equally broad and actively promoted among all players.

\section{SDGs AS A HEALTH SYSTEM}

A health system, broadly conceived, provides essential medical and public health services (centered on SDG 3) but also links health with agriculture, education, employment, energy, environment, finance, trade, transport and urban planning (embraced by the other 16 Goals), potentially with mutual benefits. Whether an intervention affects health directly or indirectly, the properties of an effective system are the same. A skilled workforce, medical technologies, infrastructure, and finance and governance mechanisms are all needed to deliver services that should be affordable, efficient, equitable, ethical, measureable, responsive, resilient, sustainable and testable. The importance of understanding how to instill these properties was neglected in the MDG era, even though they are a precondition to achieving high population coverage of technologies including diagnostics, drugs, vaccines and insecticides. Some examples of the opportunities and challenges in strengthening health systems are below.

Affordable. To provide full coverage of health services while avoiding financial hardship for patients is a major test for UHC (10). The first international conference on financing in the SDG era, held in Addis Ababa in 2015, concluded that each country should take primary responsibility for its own economic and social development, including measures to increase domestic public finance for health, principally through progressive taxation that favors the poorest (a task for HiAP), and decrease reliance on foreign aid (12). Given the scale of the task for example, to avoid out-of-pocket payments for health services that drive more than 100 million people into poverty every year - the costs are expected to be substantial. By one estimate, the governments of 67 low- and middle-income countries would need to nearly triple current spending on health between 2014 and 2030 (13).

The immediate financial challenge to poorer countries is enormous but, subject to evaluation, could bring benefits in the long run. One is to reduce dependency on unpredictable and earmarked foreign aid, thereby stimulating self-sufficiency. Another is the incentive to improve efficiency, given limited resources, by streamlining financial flows, by cutting wasteful spending, by pooling health revenues to maximize options for redistribution, and by matching funds to priority health services and populations. In addition, tackling the causes of illness upstream could deliver a double benefit: costs can in principle be shared across sectors to achieve compatible objectives, for example through joint policies on energy use, climate change, air quality and health, while saving on the costs of treating illnesses that have been avoided. In this domain, the question of how best to translate theory into practice is itself a topic for research.

The age-old adage that prevention is better than cure is not yet backed by a satisfactory case for investment. One alluring reason to revisit the options for prevention is that health risks from avoidable exposures explain a large fraction of deaths worldwide (environment $>28 \%$, behavioral $>67 \%$ ) (15) and yet, according to national health accounts, preventive and public health services command only $4 \%$ of around US\$8 trillion spent on global health annually (10). The question of how much to spend on prevention remains unresolved, in part because investments that bring health benefits, but where health is not the primary purpose (in other sectors such as agriculture, education and transport), have not been systematically quantified and so remain invisible.

Studies of disease burden have ranked causes of illness and death both in terms of health outcomes (ischaemic heart disease, cerebrovascular disease, lower respiratory infections) and preventable risks (high blood pressure, tobacco smoking, air pollution). Which list should be used to set priorities for intervention? What economic methods are preferred when comparing the returns on direct (health sector) and indirect (other sectors) investments in health, among other desirable outcomes of development?

Equitable. Inequity is not merely a numerical impediment to achieving high levels of service coverage, "leaving no one behind," it also denies individuals the right to health by compromising their freedoms and entitlements, which are goals in their own right. In the 2030 Agenda, goals for health and equity stand on common ground, and there is mutual support for approaches that foster better health among the disadvantaged (SDG 3), gender equality (SDG 5), equality within countries in general (SDG 10), and transparency, accountability and nondiscriminatory laws (SDG 16). Deeper investigations of how to exploit these linkages could accelerate recent positive trends: for example, in spite of rising income inequality within countries, there is growing evidence that inequalities in access to health services are falling, and they appear to be falling more (immunization) or less (antenatal care) quickly depending on the service provided (18). This points to the research needed to identify the financial and economic determinants of health service provision, for poorer or richer members of each population.

Although some of the barriers to health care, and therefore ways to overcome them, are unique to specific settings, there are some generalities. One is that equity and prevention often go hand in hand: for example, policies for universal education and the reduction of outdoor air pollution have widespread benefits for heath (19).

Measureable. The list of 230 agreed indicators that track progress towards the SDGs emphasizes the outcomes for development (what is to be achieved), rather than the "means of implementation" (how it will be achieved). These include financing mechanisms, the development and application of technologies, capacity building, and global and national partnerships, policies and institutions. WHO lists a subset of 42 healthrelated indicators across 11 SDGs, of which 26 measure health outcomes, 12 report on the coverage of health services, but only 4 deal with factors and functions that contribute to better outcomes (employment of health workers, foreign funding for research, domestic funding for essential health services, and emergency preparedness) (20). Moreover, the underlying causes of ill health, to which interventions must respond, are no more than implicit. Whether the agreed indicators are precisely or appropriately defined, and whether they can be serviced by data, has been much discussed. But the SDG framework presents a larger challenge to measurement and evaluation: where there is no information on the underlying causes of illness and death, or on 
the components of the response, it will be harder to explain and modify health trends. Although the number of SDG indicators is already daunting, still more data will be needed, at least to resolve selected questions. The emphasis on measurement was a success of the MDG era; the SDGs have that data-analytical heritage to build on.

Sustainable. Thirty years ago, the World Commission on Environment and Development defined development as sustainable when it "meets the needs of the present without compromising the ability of future generations to meet their own needs" (21). In general, the case for long-term investment must counter the disinclination to pay now for benefits gained in future, which are perceived to have lower value because of discounted economic returns on investment, or short political time scales. One approach, crucial to mitigating the dangers of climate change, is to blend strategies that have both short- and long-term benefits, such as cutting atmospheric pollutants that are produced jointly with greenhouse gases (22).

Testable. The case for developing and testing new technologies has been abundantly made, but given the need to forge new links within and across disciplines and sectors, health systems research, in the broadest sense, is a potential source of new means of prevention and treatment. While the efficacy of drugs, vaccines and diagnostics determined by clinical trials is usually applicable in a wide range of settings, the "means of implementation" commonly depend on local circumstances. Finding the best way to implement technologies presents a wide variety of research questions demanding imaginative study designs: to test novel legal and financial instruments, to explore ways of expanding the health workforce beyond the medical profession, to create common platforms for health delivery, among many others. Now is the time to present compelling arguments for carrying out such studies, as research funding agencies become more receptive to supporting multidisciplinary and operational research.

\section{BETTER SYSTEMS, BETTER HEALTH?}

Critics who have argued that the 2030 Agenda embraces everything in general and nothing in particular are at risk of missing a huge opportunity. The SDGs are not merely an inventory of new goals and targets, drawn up to satisfy every constituency; together, they are a stimulus to find new ways of advancing health and well-being. The idea of improving health systems to achieve
UHC is at the forefront of SDG 3, but the logic of creating better systems for health applies more widely, potentially, though not necessarily, with mutual benefits between the health goal and all other goals. The notion that the SDGs are "integrated and indivisible" has been frequently articulated but the means of capturing the benefits have not yet been fully exploited, across the spectrum of activities from research and development to policy and practice. The few examples given here focus on questions about the opportunities for primary prevention, for achieving health equity, for sharing financial investment across sectors to reach common goals, and for devising long-term solutions. With only partial answers at hand, the 2030 Agenda should be seen, not as a finished roadmap for development, but as an expanded agenda for systems research. The working hypothesis is that better systems can indeed deliver substantially better health.

\section{REFERENCES AND NOTES \\ 2. S. Kuruvilla et al., Bulletin of the World Health Organi- zation 92, 533-544 (2014). \\ 3.J. Ruducha et al., Lancet Global Health 5, e1142 (2017) \\ 4. T. Hafner, J. Shiffman,. Health policy and planning 28 , 41 (2013) \\ 6. V. Haldane et al., AIDS Care 30, 103 (2018)}

9. C. Dye, M. Raviglione, Nature 502, S13 (2013)

10. A. Sen, Harvard Public Health Review4, 1 (2015).

12. World Health Organization, "New Perspectives on Global Health Spending for Universal Health Coverage," (World Health Organization, Geneva, 2017).

13. K. Stenberg et al., Lancet Global Health 5, e875 (2017).

15. GBD Risk Factors Collaborators, Lancet 390, 1345 (2017)

16. Organisation for Economic Cooperation and Development, "Health expenditure and financing," (Organisation for Economic Cooperation and Development, Paris, 2016).

18. World Health Organization, "State of inequality: childhood immunization," (World Health Organization, Geneva, 2016).

19. M. Tobias, Lancet389, 1172 (2017)

20. World Health Organization, "World Health Statistics," (World Health Organization, Geneva, 2017).

21. World Commission on Environment and Development, "Our common future," (United Nations World Commission on Environment and Development (WCED), Oxford, 1987).

22. D. Shindell et al., Science 356, 493 (2017)

\section{Acknowledgements \\ I thank B. Aylward, J. Kutzin, T. Swift Koller, S. Kuruvilla, A. Monnot, P. Rosenberg, A. Soucat, N. Valentine and B. Williams for helpful comments and sources. The author alone is responsible for the views expressed in this pa- per, which do not necessarily represent the decisions, policy, or views of the World Health Organization.}

10.1126/science.aaq1081 